\title{
The interictal dysphoric disorder of epilepsy: legend or reality?
}

\section{Marco Mula MD PhD}

Atkinson Morley Regional Neuroscience Centre, St George's University Hospitals NHS Foundation Trust, London, United Kingdom

South West London \& St George's Mental Health Trust, London, United Kingdom

Institute of Medical and Biomedical Sciences, St George's University of London, United Kingdom

\author{
Correspondence: \\ Dr Marco Mula MD PhD \\ Atkinson Morley Regional Neuroscience Centre \\ St George's University Hospitals NHS Foundation Trust \\ Blackshaw Road \\ London SW17 0QT \\ United Kingdom \\ Tel: +442087254107 \\ FAX: +442087254591 \\ Email:mmula@sgul.ac.uk
}




\begin{abstract}
For a long time, the relationships between epilepsy and mood disorders captured the attention of clinicians and neuroscientists. The existence of a peculiar clinical presentation for mood disorders in epilepsy has been matter of debate since the early reports of Kraepelin and Bleuler. The interictal dysphoric disorder (IDD) represents the modern reinterpretation of such early observations. This paper reviews current research on this topic discussing clinical implications, phenomenological observations and directions for future research.
\end{abstract}

Key words: epilepsy, interictal dysphoric disorder, peri-ictal dysphoric syndrome, antiepileptic drugs, depression, bipolar disorder 


\section{Introduction}

For a long time the association between epilepsy and depression has been matter of debate. In his famous quotation, Hippocrates report that "melancholics ordinarily become epileptics, and epileptics, melancholics: what determines the preference is the direction the malady takes; if it bears upon the body, epilepsy, if upon the intelligence, melancholy" [1]. This ancient observation has been recently revitalized by modern epidemiological data suggesting a bidirectional relationship between epilepsy and mood disorders and between epilepsy and suicide [2-4]. Although such a bidirectional link seems to be present in several chronic conditions, for example Parkinson's disease [5], stroke [6], dementia [7], diabetes [8] and cardiovascular diseases [9], the case of epilepsy is definitely intriguing given the neurobiological underpinning shared by the two conditions [10-12].

If epilepsy and depression share a number of pathophysiological mechanisms, it is also possible that there would be a peculiar syndrome characterized by seizures and mood symptoms. Epilepsy is not a unique entity, with a number of syndromes characterized by recurrent seizures as the main element. However, behavioral symptoms are rarely considered as part of whole picture. The concept of interictal dysphoric disorder (IDD) derives from original observations of classic German psychiatrists, namely Kraepelin and Bleuler, who observed that patients with untreated epilepsy could develop a pleomorphic pattern of depressive symptoms intermixed with euphoric moods, irritability, fear and anxiety as well as anergia, pain and insomnia [13, 14]. This concept has been subsequently rejuvenated, in modern times, by Blumer who actually coined the term IDD [15]. Since its introduction, the concept of IDD has been matter of debate. The aim of this paper is discussing current research on this affectivesomatoform syndrome with special attention to clinical implications. References were identified by searches of Medline/PubMed using the key words "epilepsy", "depression", "interictal dysphoric disorder". Only papers written in English have been considered. However, the list of relevant articles was hand-searched for additional publications (e.g. book chapters or review papers) if relevant for the discussion.

\section{The non-conforming features of mood disorders in epilepsy and the concept of IDD}

A number of authors agree that up to $50 \%$ of patients with epilepsy and depression present psychiatric symptoms that are not captured by standardized classificatory systems such as DSM or ICD [16-20]. This observation might explain the high variability in epidemiological data on mood disorders in epilepsy. In fact, community-based studies report prevalence rates in the order of $20 \%-22 \%[21,22]$ but, in selected populations, such as tertiary referral centers, prevalence rates seem to be as high as $50 \%[23,24]$. Although it is plausible that such differences reflect the severity of the individual seizure disorder [25, 26], it is also possible that atypical presentations of depression are not captured by international coding systems or standardized clinical instruments.

IDD is operatively conceptualized by Blumer in eight key symptoms, grouped in three major categories, namely labile depressive symptoms (depressive mood, anergia, pain, and insomnia), labile affective symptoms (fear, anxiety), and supposedly "specific" symptoms (paroxysmal irritability, and euphoric moods) (Table 1). The latter, in particular, identify a peculiar symptom cluster characterized by periodical mood changes and outbursts of irritability and aggressive behavior. Such episodes are described as occurring without external triggers and without clouding of consciousness, beginning and ending rapidly and recurring fairly regularly in a uniform manner (every 
few days to every few months and lasting a few hours up to 2 days). Interestingly, the concept of IDD goes beyond the mood disorder per se, encompassing a spectrum of conditions with transient psychotic features or even prolonged psychotic states. In other words, Blumer suggests that interictal psychoses of epilepsy represent severe forms of IDD with prominent psychotic features [15, 27].

Although it appears evident that Blumer's description has been highly influenced by the Kraepelinian view of the relationship between mood disorders and schizophrenia, modern studies on the phenomenology of IDD and psychoses of epilepsy partly support this hypothesis. In fact, IDD seems to be closer to bipolar depression rather than to unipolar one $[20,28,29]$ and psychoses of epilepsy are characterized by a preserved personality and prominent mood symptoms [30] suggesting that psychoses of epilepsy belong to the mood spectrum rather than to the psychotic spectrum. However, the symptom cluster described in patients with IDD and epilepsy is quite peculiar and is rarely reported in psychiatric practice even in rapid cycling bipolar disorders or cyclothymia [28]. Probably for this reason, Blumer stated that IDD is unique for epilepsy [27].

\section{The concept of peri-ictal dysphoric syndrome (PDS)}

It seems now established that epileptic seizures are not just characterized by the ictal phase but a number of behavioral manifestations can occur around the ictus, either preceding or following [31,32]. Such symptoms may be subtle and difficult to identify if not specifically sought. In addition, they often fail to meet temporal criteria for mood symptoms according to DSM. It is, thus, evident that such manifestations are highly responsible for the atypical presentation of mood disorders in epilepsy.

A cross-sectional study in patients with epilepsy report that most of the pleomorphic features of IDD are related to peri-ictal symptoms [33]. In fact, among patients with a diagnosis of IDD, about 50\% present symptoms with a clear-cut relationship with epileptic seizures either presenting pre-ictally or post-ictally [33]. Premonitory symptoms are reported by one-third of patients with temporal lobe epilepsy, usually preceding secondary generalized tonic-clonic seizures [34]. Prodromal mood changes may occur hours to days before a seizure and are often relieved by the convulsion [35]. A cross-sectional study in tertiary referral centers in Europe point out that around 13\% of patients may experience irritability, dysphoria or depressed mood preceding seizures [33]. As for pre-ictal symptoms, post-ictal mood changes are rarely recognized by clinicians but a case series of presurgical patients suggests that up to $18 \%$ of patients can report post-ictally at least five symptoms of depression lasting more than 24 hours and up to $22 \%$ present manic symptoms often with associated hallucinations or delusions [36]. Post-ictal anxiety seems to be even more frequent being reported by $45 \%$ of patients [36]. The median duration of symptoms ranges from 6 to 24 hours but in one third of cases, post-ictal anxiety may last 24 hours or longer.

All these data taken together have relevant implications in terms of prognosis and treatment, emphasizing the need to dissect out peri-ictal manifestations from interictal ones. It is estimated that around $12 \%$ of patients with TLE may present isolated peri-ictal dysphoric symptoms without interictal chronic symptoms [33]. In such patients a so-called peri-ictal dysphoric syndrome (PDS) can be recognized but the prognosis and treatment is that of the epileptic syndrome rather than that of a psychiatric disorder.

\section{Diagnosing IDD and PDS}


Peri-ictal symptoms are not rare but clinicians need to specifically enquire [29]. Moreover, peri-ictal symptoms may be almost indistinguishable from interictal ones, apart from duration and the close relation with seizure occurrence. It is, thus, possible, if not recognized, to overestimate psychiatric diagnosis or even misdiagnose behavioral problems in some cases. A cross sectional study of patients recruited in two epilepsy centers in Europe shows that a diagnosis of bipolar disorder may be overestimated in epilepsy if peri-ictal mood changes are not correctly identified [29]. In fact, out of the $11.8 \%$ of DSM-based diagnoses of bipolar disorder, only $1.4 \%$ could be considered as a "pure" psychiatric diagnosis because in all other cases manic/hypomanic symptoms were temporally-related to seizures occurring either post-ictally or pre-ictally [29]. A careful assessment of seizure-based behavioral manifestations should always be part of a routine assessment of patients with epilepsy and behavioral problems. In fact, the identification of patients with PDS may be relevant in terms of localizing and prognostic value. In fact, post-ictal mania seems to be associated with frontal, non-dominant involvement $[37,38]$ and is associated with a poor prognosis after surgery identifying temporal lobe "plus" patients [38]. In this regard, a specific clinical instrument for the evaluation of IDD and PDS has been developed, namely the Interictal Dysphoric Disorder Inventory (IDDI) [20]. The IDDI is a 38 items, self-report questionnaire exploring a timeinterval of 12 months. It is possible to obtain a total score and three subscale scores that mirror the three major symptom categories described by Blumer. Besides, the IDDI measures the degree of interference or distress caused by IDD symptoms. The instrument shows a good internal consistency, an acceptable sensitivity and an excellent specificity. Finally, in the Appendix to the questionnaire, six questions investigate the time course of the disorder, duration of dysphoric symptoms and their associations with seizures or antiepileptic drug therapy. A recent study investigated test-retest reliability of the IDDI showing a 50\% agreement [39]. This is something frequently seen in psychological testing as the attribute that is being measured may change between the first test and the retest; because the experience of taking the test itself can change a person's true score. (for example, completing an anxiety inventory could serve to increase a person's level of anxiety); finally because when retested, people may remember their original answer, which could affect answers on the second administration in order to minimize some symptoms. In the case of PDS, this is further complicated by the seizure itself as it is entirely possible that patients do not remember some symptoms that are part of the post-ictal state. For all these reasons a careful clinical evaluation should be part of the assessment apart from the use of clinical rating scales.

\section{Is IDD nosologically independent from other psychiatric syndromes?}

Whether IDD is a nosologically independent psychopathological construct represents the key question. That up to $50 \%$ of patients with epilepsy and mood disorders do not meet DSM criteria do not necessarily mean that all of them have IDD. A cross sectional study aimed at validating IDD against DSM criteria showed that patients with IDD fulfill criteria for a specific DSM diagnosis [20] and this has been replicated by other studies other centres [39, 40]. In addition, IDD was shown also inb patients with migraine with a fairly similar prevalence [20] suggesting that it is not disease-specific. However, studies aimed at investigating the impact of IDD on quality of life clearly showed that IDD and DSM depressive disorders have a different impact [39-41]. An interesting observation comes from a cross-sectional study aimed at investigating 
temperamental features of patients with epilepsy [42]. The authors showed that irritable and dysphoric features are more prevalent in the epilepsy population as compared to the general population. The term temperament refers to those aspects of an individual's personality that are regarded as innate rather than learned. It is now established that affective temperaments represents the main neurobiological ground on which mood disorders develop. In addition, affective temperaments influence the clinical features of a specific mood disorder in terms of both clinical and course characteristics, family history and axis I and II co-morbidities [43]. It is, thus, possible that the ultimate phenotype for mood disorders in epilepsy is shaped by an underlying dysphoric/irritable temperament making the flavor of depression in epilepsy different from that of typical endogenous depression. The identification of such clinical dimensions is not an academic exercise but has relevant implications in terms of suicidal risk [41] and post-surgical complications [45]. Whether the irritable temperament type is more prevalent in epilepsy as compared to other chronic medical conditions hasn't been established yet. Amygdala volume loss seem to be related to IDD in epilepsy [46] but whether this is typical of epilepsy only or it represents an anatomical correlates of mood dysfunction with dysphoria has to be established. Further studies aimed at investigating the relationship between temperamental features and chronic medical and neurological disorders are urgently needed.

\section{Conclusions}

The concept of IDD has been matter of discussion for a long time. However, a few points seem to be clearly established: (1) patients with epilepsy may present behavioral syndromes with atypical features compared to those currently described in wisely used classificatory systems such as DSM; (2) in several cases, such atypical features are related to peri-ictal symptoms that may not be captured or misleadingly captured by standardized clinical instruments adopted outside epilepsy; (3) peri-ictal symptoms are, by definition, typical of epilepsy, and as such, epilepsy-specific behavioral syndromes are plausible.

Further studies are needed in order to identify a peculiar clinical phenotype and its clinical characteristics. Epilepsy still today represents a privileged window into the neurobiology of behavior and mood.

\section{Acknowledgments and disclosures}

The author is currently serving as Associate Editor of Epilepsy \& Behavior.

\section{References}

[1] Temkin N. The falling sickness. Baltimore: The John Hopkins Press; 1971.

[2] Hesdorffer DC, Hauser WA, Annegers JF, Cascino G. Major depression is a risk factor for seizures in older adults. Ann Neurol 2000;47: 246-9.

[3] Hesdorffer DC, Hauser WA, Olafsson E, Ludvigsson P, Kjartansson O. Depression and suicide attempt as risk factors for incident unprovoked seizures. Ann Neurol 2006;59: 35-41. 
[4] Hesdorffer DC, Ishihara L, Mynepalli L, Webb DJ, Weil J, Hauser WA. Epilepsy, suicidality, and psychiatric disorders: a bidirectional association. Ann Neurol 2012;72: 184-91.

[5] Menza M, Dobkin RD, Marin H, Mark MH, Gara M, Buyske S, Bienfait K, Dicke A. The impact of treatment of depression on quality of life, disability and relapse in patients with Parkinson's disease. Mov Disord 2009;24: 1325-32.

[6] Thomas AJ, Kalaria RN, O'Brien JT. Depression and vascular disease: what is the relationship? J Affect Disord 2004;79: 81-95.

[7] Panza F, D'Introno A, Colacicco AM, Capurso C, Del Parigi A, Caselli RJ, Frisardi V, Scapicchio P, Chiloiro R, Scafato E, Gandin C, Vendemiale G, Capurso A, Solfrizzi V. Temporal relationship between depressive symptoms and cognitive impairment: the Italian Longitudinal Study on Aging. J Alzheimers Dis 2009;17: 899-911.

[8] Pan A, Lucas M, Sun Q, van Dam RM, Franco OH, Manson JE, Willett WC, Ascherio A, Hu FB. Bidirectional association between depression and type 2 diabetes mellitus in women. Arch Intern Med 2010;170: 1884-91.

[9] Lippi G, Montagnana M, Favaloro EJ, Franchini M. Mental depression and cardiovascular disease: a multifaceted, bidirectional association. Semin Thromb Hemost 2009;35: 325-36.

[10] Kanner AM. Mood disorder and epilepsy: a neurobiologic perspective of their relationship. Dialogues Clin Neurosci 2008;10: 39-45.

[11] Kanner AM. Depression and epilepsy: A bidirectional relation? Epilepsia 2011;52 Suppl 1: 21-7.

[12] Richerson GB, Buchanan GF. The serotonin axis: Shared mechanisms in seizures, depression, and SUDEP. Epilepsia 2011;52 Suppl 1: 28-38.

[13] Kraepelin E, Diefendorf AR. Clinical psychiatry (1907). Delmar, N.Y.: Scholars' Facsimiles and Reprints; 1981.

[14] Bleuler E. Textbook of psychiatry. New York: The Macmillan Co; 1924.

[15] Blumer D. Dysphoric disorders and paroxysmal affects: recognition and treatment of epilepsy-related psychiatric disorders. Harv Rev Psychiatry 2000;8: 8-17.

[16] Kanner AM, Kozak AM, Frey M. The Use of Sertraline in Patients with Epilepsy: Is It Safe? Epilepsy Behav 2000;1: 100-105.

[17] Mendez MF, Cummings JL, Benson DF. Depression in epilepsy. Significance and phenomenology. Arch Neurol 1986;43: 766-70.

[18] Krishnamoorthy ES, Trimble MR, Blumer D. The classification of neuropsychiatric disorders in epilepsy: a proposal by the ILAE Commission on Psychobiology of Epilepsy. Epilepsy Behav 2007;10: 349-53.

[19] Mula M. Epilepsy: Bidirectional link between epilepsy and psychiatric disorders. Nat Rev Neurol 2012;8: 252-3.

[20] Mula M, Jauch R, Cavanna A, Collimedaglia L, Barbagli D, Gaus V, Kretz R, Viana M, Tota G, Israel H, Reuter U, Martus P, Cantello R, Monaco F, Schmitz B. Clinical and psychopathological definition of the interictal dysphoric disorder of epilepsy. Epilepsia 2008;49: 650-6.

[21] Edeh J, Toone BK. Antiepileptic therapy, folate deficiency, and psychiatric morbidity: a general practice survey. Epilepsia 1985;26: 434-40.

[22] Tellez-Zenteno JF, Patten SB, Jette N, Williams J, Wiebe S. Psychiatric comorbidity in epilepsy: a population-based analysis. Epilepsia 2007;48: 2336-44.

[23] Victoroff JI, Benson F, Grafton ST, Engel J, Jr., Mazziotta JC. Depression in complex partial seizures. Electroencephalography and cerebral metabolic correlates. Arch Neurol 1994;51: 155-63. 
[24] Ring HA, Moriarty J, Trimble MR. A prospective study of the early postsurgical psychiatric associations of epilepsy surgery. J Neurol Neurosurg Psychiatry 1998;64: 601-4.

[25] Jacoby A. Psychosocial functioning in people with epilepsy in remission and the outcomes of antiepileptic drug withdrawal. [electronic resource]. In: University of Newcastle upon Tyne; 1995.

[26] O'Donoghue MF, Goodridge DM, Redhead K, Sander JW, Duncan JS. Assessing the psychosocial consequences of epilepsy: a community-based study. Br J Gen Pract 1999;49: 211-4.

[27] Blumer D, Montouris G, Davies K. The interictal dysphoric disorder: recognition, pathogenesis, and treatment of the major psychiatric disorder of epilepsy. Epilepsy Behav 2004;5: 826-40.

[28] Mula M. The clinical spectrum of bipolar symptoms in epilepsy: a critical reappraisal. Postgrad Med 2010;122: 17-23.

[29] Mula M, Schmitz B, Jauch R, Cavanna A, Cantello R, Monaco F, Trimble MR. On the prevalence of bipolar disorder in epilepsy. Epilepsy Behav 2008;13: 658-61.

[30] Trimble MR. The psychoses of epilepsy. New York: Raven Press; 1991.

[31] Mula M, Monaco F. Ictal and peri-ictal psychopathology. Behav Neurol 2011;24: 21-5.

[32] Mula M, Monaco F. Can patients with epilepsy predict their seizures? Neurology 2007;69: 494; author reply 494.

[33] Mula M, Jauch R, Cavanna A, Gaus V, Kretz R, Collimedaglia L, Barbagli D, Cantello $\mathrm{R}$, Monaco F, Schmitz B. Interictal dysphoric disorder and periictal dysphoric symptoms in patients with epilepsy. Epilepsia 2010;51: 1139-45.

[34] Gaitatzis A, Trimble MR, Sander JW. The psychiatric comorbidity of epilepsy. Acta Neurol Scand 2004;110: 207-20.

[35] Blanchet P, Frommer GP. Mood change preceding epileptic seizures. J Nerv Ment Dis 1986;174: 471-6.

[36] Kanner AM, Soto A, Gross-Kanner H. Prevalence and clinical characteristics of postictal psychiatric symptoms in partial epilepsy. Neurology 2004;62: 708-13.

[37] Nishida T, Kudo T, Inoue Y, Nakamura F, Yoshimura M, Matsuda K, Yagi K, Fujiwara T. Postictal mania versus postictal psychosis: differences in clinical features, epileptogenic zone, and brain functional changes during postictal period. Epilepsia 2006; 47: 2104-14.

[38] Barba C, Barbati G, Minotti L, Hoffmann D, Kahane P. Ictal clinical and scalp-EEG findings differentiating temporal lobe epilepsies from temporal 'plus' epilepsies. Brain 2007;130: 1957-67.

[39] Amiri M, Hansen CP. The interictal dysphoric disorder in patients with epilepsy: A doubtful disorder lacking diagnostic tools. Seizure. 2015;24:70-6.

[40] Suda T, Tatsuzawa Y, Mogi T, Yoshino A. Interictal dysphoric disorder in patients with localization-related epilepsy: Diagnostic relationships with DSM-IV psychiatric disorders and the impact of psychosocial burden. Epilepsy Behav. 2016;54:142-7.

[41] Andrade-Machado R, Ochoa-Urrea M, Garcia-Espinosa A, Benjumea-Cuartas V, Santos-Santos A. Suicidal risk, affective dysphoric disorders, and quality-of-life perception in patients with focal refractory epilepsy. Epilepsy Behav. 2015;45:254-60.

[42] Erdoğan Taycan S, Taycan O. Affective temperament profiles and clinical correlates in patients with epilepsy: a link from mood disorders. J Affect Disord. 2014;164:1-4.

[43] Perugi G, Toni C, Maremmani I, Tusini G, Ramacciotti S, Madia A, Fornaro M, Akiskal HS. The influence of affective temperaments and psychopathological traits on the 
definition of bipolar disorder subtypes: a study on bipolar I Italian national sample. J Affect Disord. 2012;136(1-2):e41-9.

[45] Hellwig S, Mamalis P, Feige B, Schulze-Bonhage A, van Elst LT. Psychiatric comorbidity in patients with pharmacoresistant focal epilepsy and psychiatric outcome after epilepsy surgery. Epilepsy Behav. 2012;23(3):272-9.

[46] Elst LT, Groffmann M, Ebert D, Schulze-Bonhage A. Amygdala volume loss in patients with dysphoric disorder of epilepsy. Epilepsy Behav. 2009;16(1):105-12 
Table 1. Symptom clusters for interictal dysphoric disorder.

Labile depressive symptoms

Depressed mood

Anergia

Pain

Insomnia

Labile affective symptoms

Fear

Anxiety

Specific symptoms

Mood swings

Paroxysmal irritability

Euphoric moods 